\title{
Immunohistochemical Study of CA 125 in Surface Epithelial Tumors of Ovary in Correlation with Serum Levels
}

\author{
Karishma Pillarisetty* and Savithri Ravindra \\ Department of Pathology, Kempegowda Institute of Medical Sciences, \\ Banashankari 2nd Stage, Bangalore, Karnataka, India. 560070
}

\begin{abstract}
Background: Ovarian carcinoma is the 4th leading cancer among women in India.Primary ovarian neoplasms exhibit a wide range of histopathological patterns and tumors with epithelial differentiation are most frequent. Among malignant tumors, most common histological type is serous adenocarcinoma whose diagnosis is established in advanced stages of disease in approximately $75 \%$ of patients.

The most widely used tumor marker in ovarian cancer, often considered "gold standard" is Cancer Antigen125. Cancer Antigen 125 is a high molecular weight glycoprotein which is raised in approximately $90 \%$ of patients with advanced epithelial ovarian cancer.

Methods: A 2 year prospective study included 81 cases of ovarian neoplasms with surface epithelial differentiation. The specimens were fixed in $10 \%$ formalin, routinely processed. Sections of 4-5 microns thickness were obtained from the paraffin block and stained with Hematoxylin \& Eosin. The tumors were categorised according to WHO classification. Immunohistochemical analysis of Cancer Antigen 125 was done in all malignant \& borderline tumors.
\end{abstract}

Result: A total of 81 cases were studied. There were 15 cases with elevated serum Cancer Antigen 125 levels. Of these 8 showed positive tissue expression. The sensitivity of serum Cancer Antigen 125 was $68.75 \%$ \& its specificity was $93.8 \%$.

Conclusion: Serum Cancer Antigen 125 is elevated in ovarian tumors especially in malignant surface epithelial tumors \& more commonly in serous cystadenocarcinoma.There was a good correlation between serum levels \& tissue expression of Cancer Antigen 125.

Keywords: Ovarian Cancer; Ca 125; Immunohistochemistry, Cancer Antigen 125.

\section{Introduction}

Ovarian tumors account for $5 \%$ of all the malignancies.

${ }^{[1]}$ Epithelial neoplasms being most frequent. ${ }^{[2]}$ Among malignant tumors, most common histological type is serous adenocarcinoma whose diagnosis is established in advanced stages of disease in approximately $75 \%$ of patients. ${ }^{[3]}$ The most widely used tumor marker in ovarian cancer, often considered gold standard is Cancer Antigen 125 (CA125) which is a high molecular weight glycoprotein containing 2 major antigenic domains, namely A \& B, which bind the monoclonal antibodies OC125 and MII respectively. ${ }^{[4]}$

When stratified by disease stage, elevated levels were found in more than $90 \%$ of patients with advanced stage but in only $50 \%$ of patients with Stage I disease. In addition, elevated levels of CA125 are more strongly associated with serous, rather than mucinous tumors. ${ }^{[5]}$

\section{Materials and Methods}

A prospective study was conducted for a period of 2 years with approval from institutional ethical committee. Ovarian mass lesions with surface epithelial differentiation \& with pre-operative serum CA 125 levels, were included. Those cases without pre-operative serum CA 125 were excluded.
The specimens received were fixed in $10 \%$ buffered formalin \& routinely processed. Blocks were made \& sections of 4 microns thick were cut. These sections were then stained with $H \& E$ \& categorised according to the WHO classification for ovarian neoplasms.

A separate bit was taken for immunohistochemical studies. These slides were stained for CA 125 using Dako antibody. Positive staining was observed as brown membranous \& cytoplasmic staining. Immunostaining was scored semiquantitatively using a modified histoscore method based on staining intensity \& percentage of positive tumor cells. Each stained section was quantified as-

$0=$ negative or $<5 \%$ positivity of tumor cells

$1+=$ mild positivity in $5-30 \%$ of isolated tumor cells.

$2+=$ moderate positivity in $30-80 \%$ of tumor cells

$3+=$ intense positivity in $>80 \%$ of tumor cells. ${ }^{[6,7]}$

The histopathological findings were correlated with the serum levels \& tissue expression of CA 125. 


\section{Result}

The study included 81 cases of ovarian surface epithelial neoplasms. The age of the patients ranged between 10 to 71 years with the peak age for ovarian tumors being 41 to 50 years. The most common presenting symptom was pain abdomen (49 patients) followed by mass abdomen (22 patients). Three patients presented with ascites. Left sided ovarian masses $(50.6 \% \mathrm{n}=41$ cases $)$ were slightly more common compared to the Right sided $(41.9 \% \mathrm{n}=$ 34 cases). Majority of the cases were unilateral ( 75 cases, $92.5 \%$ ). There were 6 cases with bilateral involvement. Of the 75 unilateral cases, 62 cases were benign, 4 were borderline and 9 were malignant. Among 6 bilateral cases, 3 were benign and 3 malignant.

Grossly the size of the ovarian tumors ranged from 1.5 to $30 \mathrm{cms}$. Most of the tumors were cystic (66/81 cases) [Fig. 1], 6 cases were solid in consistency [Fig 2] \& 10 cases with solid cystic consistency. There was one case with bilateral involvement where the tumor was purely solid on one side \& cystic on the other.

The commonest tumor was serous cystadenoma [Fig 3A] followed by mucinous cystadenoma [Fig 3B]. There were 2 cases of brenner tumor [Table 1]. The serum CA 125 levels in these patients ranged from 6.31 to $4117 \mathrm{U} / \mathrm{ml}$, reading $>35 \mathrm{U} / \mathrm{ml}$ was considered elevated. There were 15 cases with raised levels of serum CA 125, comprising of 8 malignant, 3 borderline and 4 benign cases. Of the 81 cases, 12 were malignant and 4 borderline ovarian tumors.
Out of these 16 (borderline \& malignant cases), 11 showed raised serum CA 125 levels. The sensitivity of serum CA 125 was $68.75 \%$ \& its specificity was $93.8 \%$.

All the cases with elevated serum CA 125 levels \& all the malignant tumors were taken for IHC along with positive $\&$ negative controls. Fallopian tube was taken as positive control. Two benign serous cystadenoma with normal serum CA 125 levels were taken as negative control. These included 20 cases of surface epithelial origin, which had 14 serous, 5 mucinous, 1 Brenner $\& 1$ case with endometrioid differentiation. Nine out of these 20 cases showed positive expression on the tumor tissue. Of the 9 cases that showed positive expression, 7 were serous cystadenocarcinoma [Fig 4A], 1 atypical proliferative serous tumor [Fig 4B] \& 1 mucinous carcinoma. [Table 2] IHC was negative in all the 4 benign serous tumors which showed increased serum CA 125 levels.

\section{Discussion}

Ovaries are a pair of female reproductive organs. Ovarian disorders have the potential to affect a woman's fertility and hormonal balance \& can range from a small cyst to ovarian cancer. Ovarian neoplasm is the $4^{\text {th }}$ most common gynaecological malignancy affecting the women in India. ${ }^{[1]}$

CA125 is a pre-operative serum marker used for early detection of ovarian cancer and has been classified as a risk \& diagnostic marker. Studies have shown that CA125 is a predictive marker that is known to increase in malignant surface ovarian tumors. ${ }^{[8]}$

Table 1: Histological Diagnosis \& Frequency of Surface Epithelial Tumors.

\begin{tabular}{|c|c|c|c|c|}
\hline Differentiation & Benign & Borderline & Malignant & Total \\
\hline & 46 & 2 & 36 & 15 \\
\hline Mucinous & 10 & 2 & 0 & 5 \\
\hline Endometrioid & 5 & 0 & 1 & 3 \\
\hline Brenner & 2 & 0 & 0 & 2 \\
\hline
\end{tabular}

Table 2: Histological correlation of IHC with serum CA 125 levels.

\begin{tabular}{|c|c|c|c|}
\hline HISTOPATHOLOGY (n= CASES) & CA 125 <35U/mI & CA 125 >35U/mI & $\begin{array}{c}\text { POSITIVE EXPRESSIONF } \\
\text { CA 125 ON TISSUE }\end{array}$ \\
\hline Benign serous cystadenoma & 40 & 4 & 0 \\
\hline Borderline serous tumor & 0 & 2 & 1 \\
\hline Borderline mucinous tumor & 1 & 7 & 7 \\
\hline Serous carcinoma & 1 & 1 & 1 \\
\hline Mucinous carcinoma & 2 & 0 & 0 \\
\hline Endometrioid carcinoma & 1 & & 7 \\
\hline
\end{tabular}




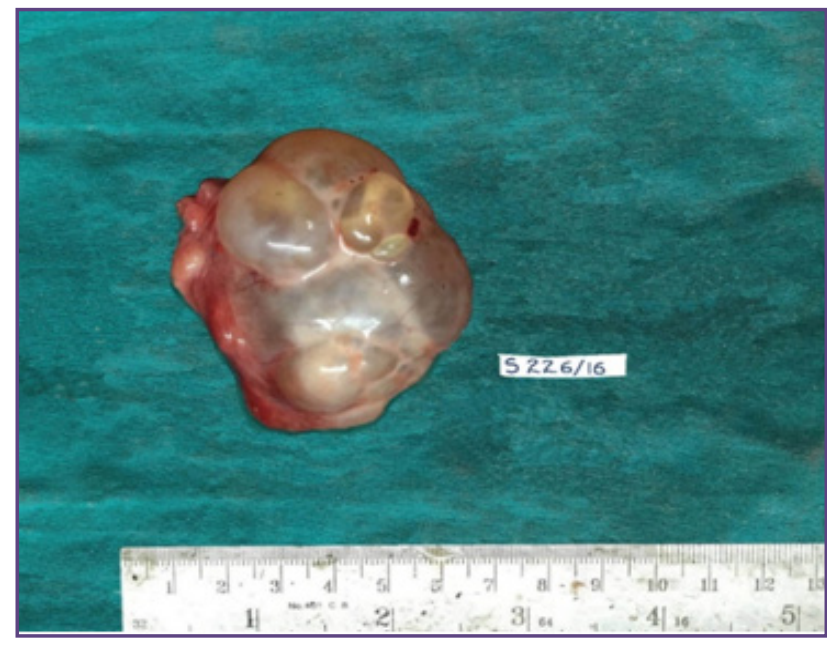

Fig. 1: Gross photograph showing cystic ovarian tumor.

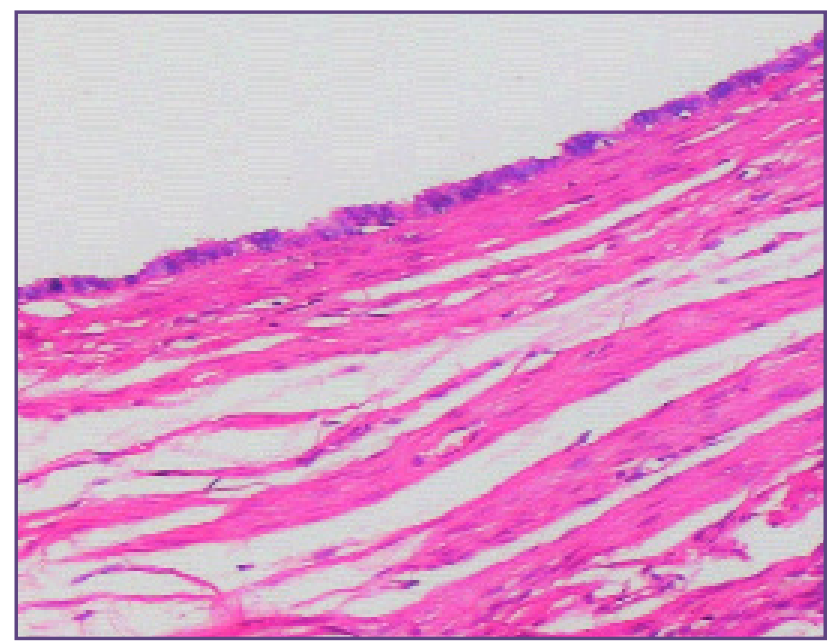

Fig. 3A: Microscopy of benign serous tumor [Hematoxylineosin X100]

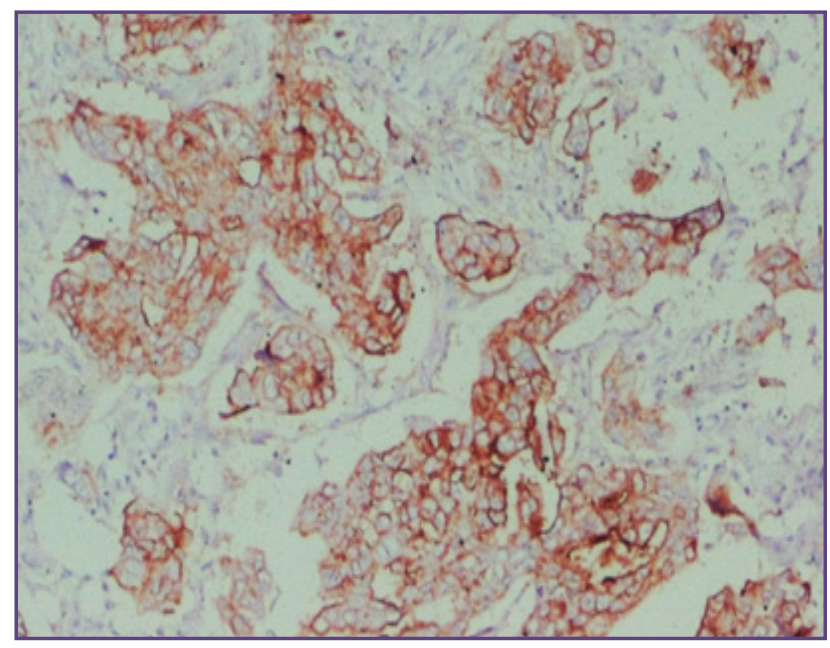

Fig. 4A: IHC CA 125 showing positive staining in serous cystadenocarcinoma [ IHC X40].

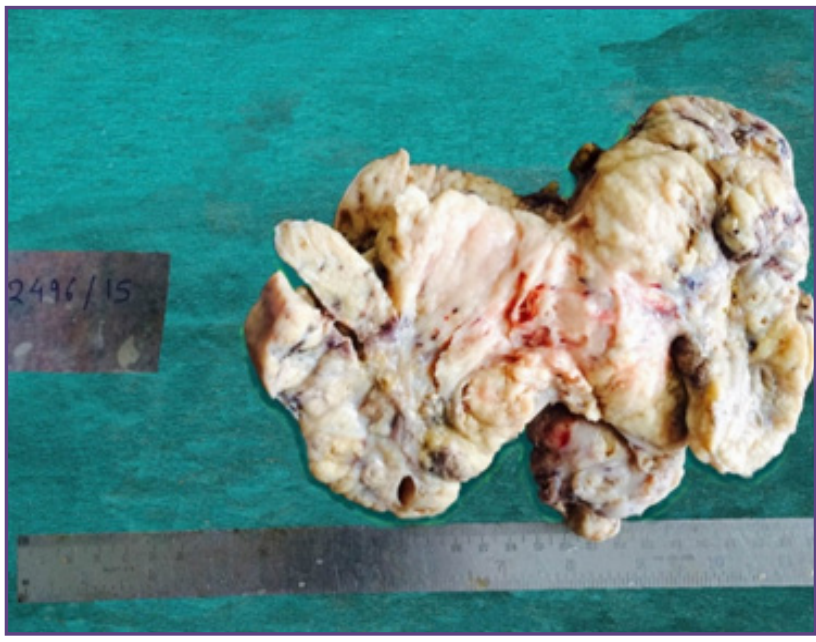

Fig. 2: Gross photograph of solid ovarian tumor.

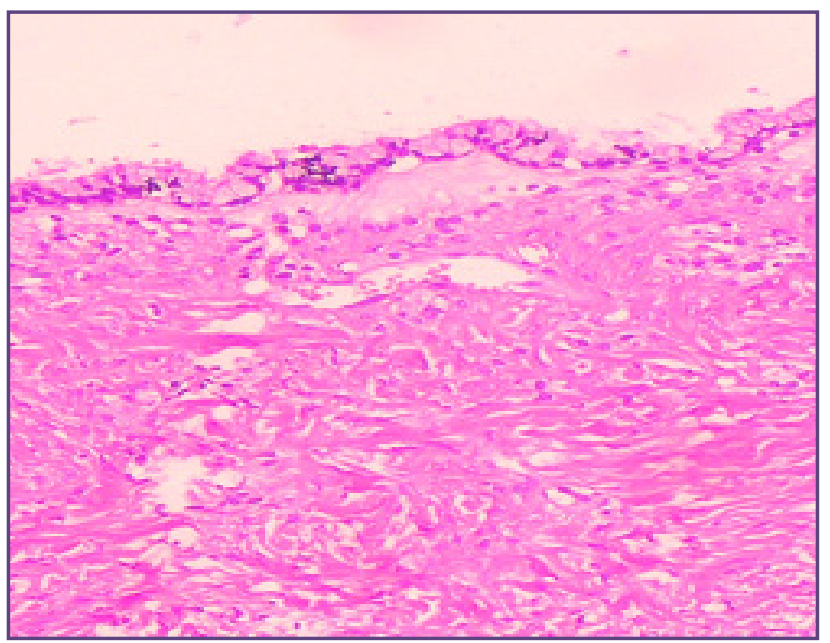

Fig. 3B: Microscopy of benign mucinous tumor [Hematoxylin - eosin X100].

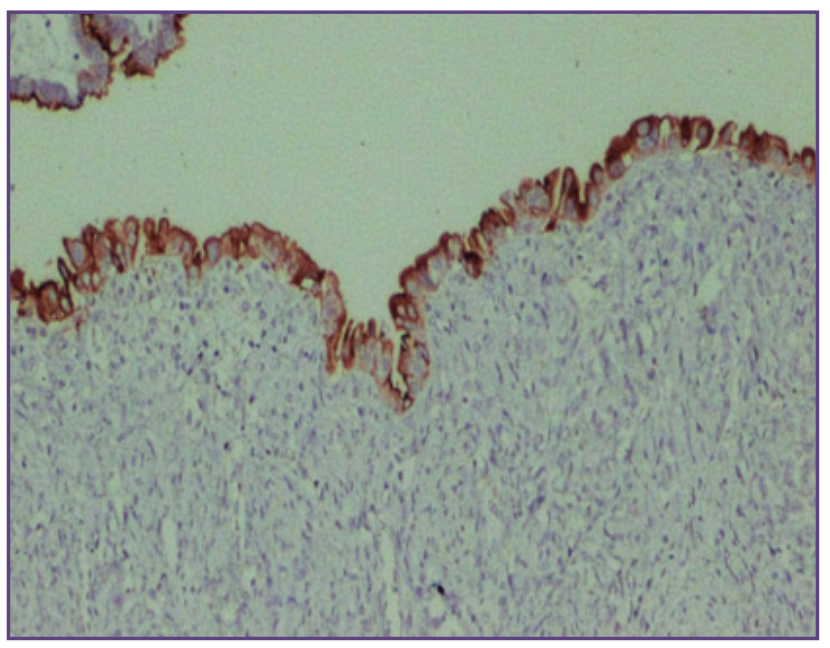

Fig. 4B: IHC CA 125 showing positive staining in borderline serous tumor [ IHC X40]. 
In the present study we found ovarian tumors between the age group of 10 to 71 years. Ghartimagar D et al noted age ranging from 13 to 90 years in their study of 310 cases of ovarian tumors. ${ }^{[9]}$ Chhanda Das et al reported 5 to 62 years in their study of 50 cases of ovarian neoplasms which was similar to other studies. ${ }^{[6,10]}$

Most benign tumors occurred in the age range of 21 to 50 years that was consistent with other studies. ${ }^{[1]}$ The peak incidence for malignancy in surface epithelial tumors was between 41 to 50 years of age. This observation was similar to the study by Bhagyalakshmi A et al \& Mondal SK et al. ${ }^{[12,13]}$ However the age incidence in our study for borderline epithelial tumors were between 21-30 years which was a decade earlier compared to the studies by Bhagyalakshmi A et al \& Mondal SK et al. ${ }^{[12,13]}$ The present study showed that the mean age for malignancies in surface epithelial tumors was 47.36 years, that was similar to the other studies. ${ }^{[14,15]}$

Majority of the cases $(92.5 \%)$ in our study showed unilateral involvement \& only $7.4 \%$ of the cases had bilateral involvement. Four of the 18 malignant tumors $\&$ only 3 of the 65 benign tumors were bilateral. All the 4 borderline tumors were unilateral. Involvement of left ovary $(50.6 \%)$ was slightly more than right side similar to study by Pradhan A et al and was in contrast to the findings of Ajani MD et al. ${ }^{[1,16]}$ Size of the ovarian tumors vary. Some of the largest tumors reported in the literature were mucinous cystadenomas, with diameter as large as 50 $\mathrm{cms}$. Some of these mucinous tumors have been recorded to weigh as heavy as $100 \mathrm{kgs}^{[7]}$ In our study the tumor size ranged from $1.5 \mathrm{cms}$ to $30 \mathrm{cms}$. Both the largest $\&$ the smallest tumors were serous cystadenoma.

Ovarian tumors can be cystic, solid or solid cystic. Generally benign \& borderline surface epithelial tumors tend to be cystic. Majority of the benign surface epithelial tumors were cystic.

The new WHO Classification of Ovarian Cancer published in 2014 by Robert Kurman and co-authors added a new entity, the seromucinous tumor \& removed transitional cell type of ovarian cancer. ${ }^{[17]}$ Surface epithelial tumors are the commonest $\&$ amount for $2 / 3^{\text {rd }}$ of all ovarian neoplasms, with benign tumors being the most common. ${ }^{[18]}$ Among these the most common were the serous tumors (56/81 cases) that was similar to the observations made by Kar Tushar et al, Pravakar \& Maingi K. ${ }^{[19,20]}$

Serum CA 125 - CA125 antigen is a high molecular weight glycoprotein, found elevated mainly in epithelial ovarian cancers. Elevated CA 125 levels were found in 82\% of women with epithelial ovarian cancers. ${ }^{[21]}$ The frequency of elevated levels is greater in patients with serous than mucinous tumors. This can be due to the secretions of various type of mucins that may alter the expression CA125 or possibly due to the inability or failure of antigen to reach the vascular compartment. ${ }^{21,22]}$

High levels of serum CA 125 are also seen in other conditions such as endometriosis, adenomyosis, fibroid, cirrhosis, renal failure, mesothelioma, breast cancer, endometrial cancer, pancreatic cancer $\&$ colon cancer. ${ }^{[8,23]}$

In the present study serum CA 125 levels were elevated in 18 of 81 patients (17.64\%). Correlating with the histopathological report, serum levels were elevated more commonly in malignant tumors; 7 cases of serous cystadenocarcinoma, 1 case of mucinous cystadenocarcinoma. Two cases of borderline serous tumor \& one case of borderline mucinous tumor also had elevated serum levels. There were 4 cases of benign serous cystadenoma that had elevated serum levels. Eva Kolwijck et al found that preoperative CA-125 levels are elevated more frequently in patients with APST compared to patients with APMT. ${ }^{[24]}$

V. Thakur et al noted very high CA 125 levels in the serum in endometrioid carcinoma. ${ }^{[25]}$ We had one case of endometrioid carcinoma which did not show increased serum levels nor tissue expression. The present study had sensitivity of CA 125 in serum of $68.75 \%$ which correlated well with other studies. There were 5 false negative cases may be due to lacking production of CA 125 in the tumor cells, but it may also be explained by impaired release of the antigen from the tumor. ${ }^{[26]}$ Present study had a specificity of $93.5 \%$. Serum CA 125 levels were seen elevated in 4 benign serous cystadenomas. These findings correlated well with the studies done by Padhariya BB et al, Jacob et al with their specificity being $83.33 \%$ \& $92 \%$ respectively. ${ }^{[27,28]}$ Study done by Chhanda Das et al had showed 19 (38\%) cases with elevated serum levels, two of which were benign. ${ }^{[6]}$

Immunohistochemistry CA 125- Preoperative serum CA 125 levels were elevated in 15 cases. Immunohistochemistry was performed in all the malignant \& borderline tumors $\&$ also in those cases where serum levels were elevated. Out of the 8 cases of serous cystadenocarinoma, 6 cases $(75 \%)$ had significant expression of CA 125 , but of the 3 cases of mucinous cystadenocarcinoma, only 1 $(33.33 \%)$ had positive expression. A case of endometrioid carcinoma was negative. The observation was similar to the study by Kriplani D et al who noted $77 \%$ of the serous carcinomas \& $25 \%$ of mucinous carcinomas with significant expression. ${ }^{[7]}$ 
The 4 borderline tumors comprised of two serous \& two mucinous tumors. Out of these 4 borderline tumors, only 1 case of serous borderline tumor showed positive tissue expression.

Similarly Kolwijck et al also found immunohistochemical CA-125 expression to be present in $90 \%$ of APST compared with $6 \%$ of APMT. ${ }^{[25]}$ The negative expression in tumors may be possibly due to the loss of CA 125 in tissue during formalin fixation or processing. Hence ample sampling of tissue may help reduce the disparity. ${ }^{[5,6]}$ Immunohistochemistry for CA 125 may be useful in poorly differentiated serous carcinoma in establishing the diagnosis.

\section{Conclusion}

Preoperative serum CA 125 levels are increased mainly in malignant tumors especially in the serous type. CA 125 in serum can increase non specifically but the tissue expression is more specific. Increased serum levels \& tissue expression correlate well in serous carcinomas.

Immunohistochemistry for CA 125 may be useful in poorly differentiated serous carcinoma in establishing the diagnosis. It also reacts with the malignant cells in undifferentiated carcinoma helping in diagnosing the ovarian origin of the tumor.

Estimation of pre-operative serum CA 125 is an easy, economical test which has a predictive value in ovarian tumors.

\section{Abbrevation}

CA 125 - Cancer antigen 125

IHC - Immunohistochemistry

\section{Acknowledgements}

None

\section{Funding}

Nil

\section{Competing Interests}

No

\section{References}

1. Saranath D, Khanna A. Current Status of Cancer Burden: Global and Indian Scenario. Biomed Res J 2014;1(1):1-5.

2. Hogdall EV, Christensen L, Kjaer SK, Blaakaer J, KjaerbyeThygesen A, Gayther S, Jacobs I.J, Hogdall C.K. CA125: expression pattern, prognosis and correlation with serum CA125 in ovarian tumor patients: from the Danish "MALOVA" ovarian cancer study. Gynecol Oncol. 2007; 104:508-515.
3. Eduardo C, Lima R de, Luís TS, Lais PK. The relationship between serum levels of CA 125 and the degree of differentiation in ovarian neoplasms. J. Bras. Patol. Med. Lab. 2014 Feb; 50(1): 20-25.

4. Gupta D, Lis CG. Role of CA125 in predicting ovarian cancer survival-a review of the epidemiological literature. J Ovarian Res. 2009;2:13.

5. Devan SM, Pailoor J, Sthaneshwar P, Narayanan V. Pattern of tissue expression of CA-125 and HE4 in primary epithelial ovarian tumours and correlation with serum CA-125 levels. Asian Pac J Cancer Prev. 2013;14(8):4545-8.

6. Das C, Mukhopadhyay M, Ghosh T, Saha AK, Sengupta M. Correlation of Cytohistlogical Expression and Serum Level of Ca125 in Ovarian Neoplasm. Journal of Clinical and Diagnostic Research. 2014 Mar; Vol-8(3): 41-43.

7. Kriplani D, Patel MM.Immunohistochemistry: A diagnostic aid in differentiating primary epithelial ovarian tumors and tumors metastatic to the ovary. South AsianJ Cancer. 2013 Oct-Dec; 2(4): 254-258.

8. Scholler N, Urban N. CA 125 in Ovarian Cancer. Biomark Med. 2007 December;1(4): 513-523.

9. Ghartimagar D, Ghosh A, KC G, Ranabhat S, Talwar OP. Surface epithelial tumors of ovary - an analysis in a tertiary referral hospital. Journal of Pathology of Nepal.2013; Vol. 3: 397-402.

10. GG Swamy, N Satyanarayana. Clinicopathological analysis of ovarian tumors - A study on five years samples. Nepal Med Coll J. 2010; 12(4): 221-223.

11. Pradhan A, Sinha AK, UpretiD.Histopathological patterns of ovarian tumors at BPKIHS. HealthRenaissance.MayAugust 2012; Vol 10(No. 2): 87-97.

12. Bhagyalakshmi A, Sreelekha A, Sridevi S, Chandralekha J, Parvathi G, Venkatalakshmi A. Prospective study of histopathological patterns of ovarian tumours in a tertiary care centre. Int J Res Med Sci. 2014 May;2(2):448-456.

13. Mondal SK, Banyopadhyay R, Nag DR, Roychowdhury S, Mondal PK, Sinha SK. Histologic pattern, bilaterality and clinical evaluation of 957 ovarian neoplasms: A10-year study in a tertiary hospital of eastern India. J Can Res Ther. 2011;7:433-7.

14. Wasim T, Majrroh A, Siddiq S. Comparison of clinical presentation of Benign and Malignant Ovarian Tumours. J Pak Med Assoc. 2009;59(1):18-21.

15. Ahmad Z, Kayani N, Hasan SH, Muzaffar S, Gill MS. Histopathological pattern of ovarian neoplasm. J Pak Med Assoc, 2000; 50: 416-9.

16. Ajani MA, Aramide KO, Salami A, Okolo CA. Histopathological pattern of Primary Ovarian Neoplasms in South Western Nigeria. Jos Journal of Medicine. July 2016; Vol 10(1): 1-8.

17. Meinhold-Heerlein I, Fotopoulou C, Harter P, Kurzeder C, Mustea A, Wimberger P, et al. The new WHO classification 
of ovarian, fallopian tube, and primary peritoneal cancer and its clinical implications. Arch Gynecol Obstet. 2016 Jun;293(6):1367.

18. Mills SE, Greenson JK, Hornick JL, Longacre TA, Reuter VE. Sternberg's Diagnostic Surgical Pathology. 6th ed. Vol 2. China: Wolters Kluwer; 2015.p.2536-2613.

19. Tushar K, Asaranti K, Mohapatra PC. Intra-operative cytology of ovarian tumours. J ObstetGynecol India. July/ August 2005; Vol. 55(4):345-349.

20. Pravakar BRE, Maingi K. Ovarian tumours- Prevalence in Punjab- A study of 636 cases. Ind J Pathol Microbiol.1989; 32:276-81.

21. Einhorn N, Bast RC, Knapp RC, Tjernberg B, Zurawski VR. Preoperative Evaluation of Serum CA 125 Levels in Patients with Primary Epithelial Ovarian Cancer. Obstet Gynecol. 1986; 67(3): 414-416.

22. Morales-Vasquez F, Pedernera E, Obregon JR, Basave HNL, Gomora MJ, CarlonE, et al. High levels of pretreatment CA125 are associated to improved survival in high grade serous ovarian carcinoma. J Ovarian Res. 2016; 9:41.
23. Bista KDB. Very High CA 125 due to Non-neoplastic Lesion of Ovary. NJOG.2012 Jul-Dec; 7(2):52-54.

24. Kolwijck E, Thomas CM, Bulten J, Massuger LFAG. Preoperative CA-125 levels in 123 patients with borderline ovarian tumors: a retrospective analysis and review of the literature. Int J Gynecol Cancer. 2009;19(8):1335-1338.

25. Thakur V, Anand AK, Mukherjee U, Ghosh D. Determination of Cancer Antigen 125 in Ovarian Carcinoma. Indian J of ClinBiochem. 2003; 18(2): 27-33.

26. O. Mogensen, B. Mogensen, A. Jakobsen, A. Sell. Preoperative Measurement of Cancer Antigen 125 (CA $125)$ in the Differential Diagnosis of Ovarian Tumors.Acta Oncologica. 1989; 28(4): 471-473.

27. Padhariya BB, Chavda J, Popat VC. Ca-125 Marker Study with Histological Correlation in Ovarian Tumor. IJAR. July 2015; Vol 5(7): 507-8.

28. Jacobs IJ, Skates S, Davies AP, WoolasRP, Jeyerajah A, Weidemann P. et al. Risk of diagnosis of ovarian cancer after raised serum CA 125 concentration: a prospective cohort study. Arch Iran med. 2010;13(3): 203-208.

*Corresponding author:

Dr. Karishma Pillarisetty, H. No 50, Parvathi, 40th Cross, 8th Block, Jayanagar, Bangalore Karnataka, India 560082

Phone: +91 9900082228

Email: karishmapillarisetty@gmail.com

Date of Submission : 24/01/2020

Date of Acceptance : 25/06/2020

Financial or other Competing Interests: None.

Date of Publication : 29/07/2020 